\author{
A. M. Oudesluys-Murphy \\ H. L. M. van Straaten \\ R. Bholasingh \\ G. A. van Zanten
}

\title{
Neonatal hearing screening
}

Received: 5 May 1995

Accepted: 9 December 1995

A. M. Oudesluys-Murphy (ख)

R. Bholasingh

Department of Paediatrics,

Zuiderziekenhuis, 3075 EA Rotterdam,

The Netherlands

Tel.; 010-2903000

Fax: 010-2903407

H. L. M. van Straaten

Department of Neonatology,

Sophia Hospital, Zwolle, The Netherlands

G. A. van Zanten

Department of Audiology,

Erasmus University,

University Hospital Sophia-Dijkzigt,

Rotterdam, The Netherlands
Abstract Severe congenital hearing impairment is an important handicap affecting $0.1 \%$ of live-born infants and $1 \%-2 \%$ of graduates of Neonatal Intensive Care Units. The prognosis for intellectual, emotional, language and speech development in the hearing-impaired child is improved when the diagnosis is made early and intervention is begun before the age of 6 months. The usual age at diagnosis of hearing impairment is at least 18-30 months (or even later in cases of less severe hearing impairment) where there are no screening programmes. When screening is carried out using distraction methods at the age of approximately 9 months some hearing-impaired infants are missed and those discovered are at least 1518 months before intervention begins. Neonatal screening could give hearing-impaired children the best chances for optimal care and development. Universal neonatal hearing screening is necessary, because, when neonatal hearing screening is restricted to high risk groups $30 \%-$ $50 \%$ of infants with hearing loss are not discovered. The methods available for neonatal hearing screening are discussed in this paper.

Conclusion 6 our view automated measurement of auditory brainstem responses is the most valuable. method for univer sal neonatal hear11ng screening.

Key words Hearing impairment . Neonatal hearing screening . Auditory brainstem responses

Abbreviations $A B R$ auditory brainstem responses $\cdot A R C$ auditory response cradle $\cdot d B n H L$ decibel normal hearing level $\cdot E O A E$ evoked oto-acoustic emissions - TEOAE transient evoked oto-acoustic emissions

\section{Introduction}

Normal hearing during very early life is of utmost importance for laying the basis for speech and language development [42]. Hepper and Shahidullah [17] have shown that the fetus reacts to sound before birth. It is able to discriminate between different speech sounds and recognise its mother's voice from prenatal exposure [8]. Babies learn the specific sounds of the language to which they are exposed during the first 6 months of life [27].
Normal hearing in early infancy is also essential for social and emotional development. Hearing impairment leads to sensory deprivation with failure to develop communication skills which in turn leads to learning problems [11]. Even relatively mild hearing losses of 35-40 dBnHL mean that a child misses approximately $50 \%$ of normal daily conversation with all the subsequent consequences [4].

The prognosis for the hearing-impaired child is improved when the diagnosis is made as early as possible. Studies by Markides [28] show that when a hearing aid is necessary and is placed in the first 6 months of life, speech 
Table 1 Principles for screening programmes according to Wilson and Jungner [50] and their relevance to a neonatal hearing screening programme

Principles for screening programmes according to Wilson and Jungner [50].

1. "The condition sought should be an important health problem".

2. "There should be an accepted treatment for patients with recognized disease".

3. "Facilities for diagnosis and treatment should be available".

4. "There should be a recognizable latent or early symptomatic stage".

5. "There should be a suitable test or examinition".

6. "The test should be acceptable to the population".

7. "The natural history of the condition, including development from latent to declared disease, should be adequately understood".

8. "There should be an agreed policy on whom to treat as patients".

9. "The cost of case-finding (including diagnosis and treatment) schould be economically balanced in relation to possible expenditure on medical care as a whole".

10. "Case-finding should be a continuing process and not a "once and for all" project".
Relevance to a neonatal hearing screening programme.

Children with undiscovered or too late discovered hearing loss have difficulty with communication and learning which influences life-long performance and earring ability. The prevalence of congenital hearing loss is high enough to warrent screening in the neonatal period. The prevalence of congenital hearing loss is higher than other disorders (e.g. congenital hypothyroidism: 1 per 3000 births, and phenylketonuria: 1 per 15000 births) which are screened in the neonatal period [45].

The treatment consists of fitting of hearing aids where possible and education and special training for the parents and child. The method of treatment is essentially the same as that which is available and is now used when children are detected later.

These are also essentially the same as those which are now available and are used when hearing loss is detected at a later age.

In cases of congenital hearing loss this is present in the neonatal period and can be detected using the sophisticated screening methods now available. We always have to remain alert for hearing loss occurring at a later age e.g. following meningitis.

This is the case at present with reliable sophisticated screening methods which will be extensively discussed in this paper.

The screening methods presently available are non-invasive, quick and acceptable to parents and neonates.

This is quite well known in the case of congenital deafness and the consequences of late detection are fully described in many reports $[11,14,31]$.

This policy has already been defined for those infants who are discovered at a later age.

The costs of a neonatal hearing programme may not be any higher, and could possibly be lower than the costs of distraction methods at a later age. The neonatal methods available are more specific and sensitive than those in use for distraction hearing screening. Newborn babies are easy to test during natural sleep. There is almost no incidence of transient conductive loss which might cause a reduction in specificity with respect to inner ear integrity assessment. In most countries there is a higher chance of reaching all children for screening at this age than later.

A neonatal hearing screening programme should certainly be an ongoing project, as is the distraction hearing screening programme at present. and language development are better than when placed at a later age. This is supported by studies by Elliott and Armbruster [11], Greenberg et al. [14], Mc Connell and Liff [31] and Ramkalawan and Davis [37] among others.

Approximately 1 child in every 1000 live births suffers from a serious congenital hearing impairment $[7,29]$. The prevalence of hearing impairment is much higher among the population of neonatal intensive care units with figures of $1 \%$ reported in Great Britain $[7,9], 1.5 \%$ in the Netherlands [47] and $2 \%$ in the USA [6].

The usual age at diagnosis of hearing impairment is at least 18-30 months, but it can be much later in cases of less severe hearing impairment $[15,29,39,40,47]$. The report of Martin et al. [29] on European Community countries showed that more than $50 \%$ of children with bilateral hearing impairment of more than $50 \mathrm{dBnHL}$ in the better ear are still undetected by the age of 3 years. Haggard [15] reported that in many areas of Great Britain, children were at least 2 years old before hearing impairment was confirmed even though distraction screening was available. This is also the case in Mott and Emon's [32] study and in that of Robertson et al. [39] in Australia and Veen et al. [47] in the Netherlands.

The importance of early hearing screening has long been recognised. In 1993 the National Institutes of Health in the U.S.A. [33] issued a concensus statement on the 
early identification of hearing impairment in infants and young children. The panel concluded that all infants admitted to neonatal intensive care units should be screened for hearing loss prior to discharge and universal screening should be implemented for all infants within the first 3 months of life. The Joint Committee on Infant Hearing in the U.S.A. has issued regular position statements since 1982 to endorse the goal of universal detection of infants with hearing loss as early as possible. In its 1994 statement it also stated that all infants with hearing loss should be identified before 3 months of age and receive intervention by 6 months [23].

In the Netherlands, as in many other European countries, infant hearing has a high priority in preventive health care schemes and money and resources are made available for screening programmes. Hearing screening is carried out in approximately $90 \%$ of infants at the age of 9 months using distraction methods [18]. Because of the age at which screening takes place and the fact that, when indicated, completion of the screening programme involves three screening sessions at monthly intervals, it means that the child is at least 15-18 months old before the diagnosis of hearing impairment is confirmed [18]. This means that much valuable time is lost for essential development for the child and normal development of the parent-child bonding.

In view of the advantages of early identification of infants with congenital hearing loss we wished to explore the present possibilities available for neonatal hearing screening. The world literature on projects for neonatal hearing screening comes from countries where practically all births take place in hospital $[40,43,49]$. It is then relatively simple to organise a screening programme for this "captive population". However, in the Netherlands, approximately $35 \%$ of all births take place at home and of those neonates born in hospital, approximately $35 \%$ leave hospital within a few hours of birth [44]. A neonatal hearing screening programme in the Netherlands therefore needs to be flexible enough to allow screening outside the hospital for most neonates. There is an extensive network of well baby clinics where infants and toddlers are seen regularly up to school age. Hearing screening using distraction methods are carried out at these clinics by specially trained child health district nurses and doctors. Clearly this network could be the most appropriate channel for carrying out an effective neonatal hearing screening programme in the Netherlands. Infants who remain in hospital for long periods after birth will need to be screened there.

\section{Screening programmes}

A screening test is a measure which attempts to sort out apparently healthy people who probably have a disorder from those who probably do not. A feature of most screening tests is that they are safe, simple and quick to administer to large populations and they tend to be inexpensive. The test must be valid and reliable.

Principles for screening programmes have been laid down by Wilson and Jungner in a WHO Report [50] (Table 1). A neonatal hearing screening programme could adequately fulfill the demands of these principles.

\section{Hearing screening methods}

\section{Conventional auditory brainstem evoked response}

The conventional auditory brainstem evoked response is the gold standard which is used for testing hearing $[6,13$, 20]. The use of auditory brainstem responses (ABR) was first reported by Jewett et al. in 1970 [21]. The ABR reflects the activity of the auditory pathway from the distal auditory nerve to the midbrain. This is very time consuming. The technicians must be highly trained and a trained audiologist is needed to interpret the results. Due to its costs, conventional ABR testing is indicated only for special risk cases or when hearing loss is suspected. It is not suitable for large scale screening.

\section{Behavioural hearing screening}

The most simple form of behavioural screening for hearing impairment is observation by parents. Approximately $60 \%$ of deaf children are first noticed by parents or relatives and only a small proportion by a physician [39]. The results could be even better if parents are better instructed about what to look for.

\section{Distraction testing}

The form of behavioural testing which is most widely used for screening for hearing defects is distraction testing of older infants [5, 25, 39, 48]. Distraction testing has been performed in the Netherlands since $1965[1,18,46]$ using the "Ewing method" [12] at the age of 9 months. Recently a modification of this test has been developed which uses recorded sounds produced via loud speakers. This is called the Compact Amsterdam Paediatric Audiometric Screener (CAPAS). The BOEL-test is another distraction method which is popular in Scandinavia and has also been used in some parts of the Netherlands [2]. It has been recommended because of the greater information it provides about the general development.

In the Netherlands approximately $90 \%$ of infants are screened.

In a review of the results of the Ewing method it was shown that $30 \%$ needed to be retested after the first screening because of an insufficient response and $12 \%$ needed to be tested yet again. Some $7 \%$ needed to be referred for further investigation [18]. 
Table 2 Comparisons of features of some hearing screening methods

\begin{tabular}{|c|c|c|c|c|c|}
\hline & EWING & $\mathrm{ARC}$ & TEAOE & ALGO & $\mathrm{ABR}$ \\
\hline Age (months) & 9 & Term & $0-\ldots$ & $0-6$ & $0-\ldots$ \\
\hline Time (mins) & $5-30[18]$ & Few-30 & $7.2[25] / 16.6[19]$ & $14[25] / 19[36]$ & \pm 30 \\
\hline Testers & 2 & 1 & 1 & 1 & $1(2)$ \\
\hline Training & +++ & ++ & +++ & ++ & $+++t$ \\
\hline Sound treated room & + & + & + & - & \pm \\
\hline Objective/subjective & Subjective & \pm Subjective & Objective & Objective & \pm Objective \\
\hline Sound intensity & $30-35 \mathrm{~dB}$ & $85 \mathrm{~dB}$ & $26-36 \mathrm{~dB}$ & $35 \mathrm{~dB}$ & All possible \\
\hline Preterm testing & not relevant & - & + & + & + \\
\hline Sensitivity & $79.4 \%[18]$ & & $76 \%[41] / 50 \%$ [19] & $100 \%[34]$ & Gold standard \\
\hline Specificity & $97.6 \%[18]$ & & $86 \%[41] / 52 \%[19]$ & $98.7 \%[34]$ & Gold standard \\
\hline Hearing pathway & Total & Total & Preneural & $\begin{array}{l}\text { Distal auditory } \\
\text { nerve-midbrain }\end{array}$ & $\begin{array}{l}\text { Distal auditory } \\
\text { nerve-midbrain }\end{array}$ \\
\hline Suitable for child with handicap & - & - & + & + & + \\
\hline Suitable as screening method & + & + & + & + & - \\
\hline
\end{tabular}

In Great Britain distraction testing is carried out at the age of 8 months according to guidelines from the Department of Health and Social Security [10]. The first test is failed by $16.3 \%$, the second by $6.8 \%$, and $2.6 \%-6.6 \%$ need to be referred for further diagnostic testing $[5,25]$.

Distraction tests have advantages in that the whole hearing pathway is tested. However, distraction testing is difficult and unreliable for risk groups who often also have physical or mental handicaps which hamper their reactions. Criteria for the presence or absence of a reaction are somewhat subjective [39]. These tests are quite time intensive. Both the first test, and the repeat test when necessary, last approximately 5 min each and the more extensive test before referral lasts $15-20 \mathrm{~min}$ [18]. They need highly trained and experienced staff (two persons for performing the classical Ewing test) and a fairly quiet room (ambient noise level less than $30 \mathrm{~dB}$ for the Ewing test) [18]. The child is at least 12 months old before hearing impairment is diagnosed by distraction testing because of the age at starting screening and the advice to wait approximately 4 weeks before repeating the tests when there is an insufficent response (because of the possible presence of temporary middle ear problems). This means that the child is always older than 15-18 months before hearing aids are placed or other habilitation measures are begun. Valuable time is hereby lost for those children with serious congenital hearing impairment.

Distraction testing is good for detecting children with conductive hearing loss but children with severe congenital sensorineural hearing loss are mainly detected by other methods. They are either detected earlier because of parents.' suspicions concerning hearing or they are discovered at a much later age $[5,32,39]$. In the study of Robertson et al. [39], the diagnosis of congenital sensorineural hearing loss was made at a median age of 18 months even though hearing screening was available. Of the children with sensorineural hearing loss in that study who had had distraction testing, $46 \%$ had passed and of those who had been screened by questionnaire $57 \%$ had passed. In a study of preterm and low birth weight infants Veen et al. [47] found that more than $50 \%$ of the children with a severe congenital hearing impairment were undetected by their third birthday even though a screening programme was available with distraction testing at 9 months. In the study of Mott and Emond [32], only $20 \%$ of children with sensorineural hearing impairment were detected by distraction testing and some had even passed the test.

A sensitivity of $79.4 \%$ and specificity of $97.6 \%$ has been reported for the Ewing method [18] (Table 2).

\section{Automated behavioural methods}

Neonatal hearing screening can be performed using automated behavioural methods which use sound producing equipment and register movement of the infant. The Auditory Response Cradle (ARC) has been developed for screening the hearing of normal term infants. It has a pressure sensitive mattress and head-rest which monitor head movement, body activity and respiratory activity. Sounds with an intensity of $85 \mathrm{~dB}$ are presented to the baby via headphones. The ARC compares reactions to sound with reactions when no sound is being produced. The behaviour of the baby is analysed and a pass or refer outcome is given [43].

The advantages of the ARC are as follows: it tests the whole hearing pathway, is non-invasive and does not require even the application of electrodes. It is relatively easy to use and is fairly quick. In the study of Tucker and Bhatachargatt [43], the test itself took only a few minutes but 30 min were allocated per baby to transport it and get it settled. A disadvantage is that because of the loud noise intensity needed to elicit a motor response it is not possible to detect mild hearing impairment with this method. It is not suitable for screening preterm or sick neonates. 
However, it is reported to have a high detection performance for serious hearing impairment and relatively low false-positive results $(1.3 \%)$ [43].

The Crib-o-Gram is another automated behavioural screening method fairly similar to, but less sophisticated than the ARC. Its sensitivity (75\%) and specificity (71\%) are too low when compared with the ABR [35]. It is no longer in production. Due to the fact that behavioural methods - even the automated methods - remain somewhat subjective and their sensitivity and specificity are too low they are not recommended by the Joint Committee on Infant Hearing [23].

\section{Evoked oto-acoustic emissions}

Screening using evoked oto-acoustic emissions (EOAE) is a method using physiological reflexes. EOAEs are defined as acoustic energy produced in the cochlea and recorded in the outer ear canal. EOAEs are considered to be a by-product of active movements of the outer hair cells in the healthy inner ear passage which enhance the vibration caused by a sound stimulus. The added vibrational energy partially leaks out of the cochlea through the middle ear. The oto-acoustic emissions are registered by recording these sounds in the outer ear canal. EOAEs are preneural in origin. Spontaneous EOAEs are produced without an external acoustic stimulus. Transient EOAEs (TEOAEs) are detected after an acoustic stimulus has been applied $[19,25,27,30,41,51]$. Depending on the stimulus used TEOAEs can be detected in up to $98 \%$ of humans with normal hearing and are absent when there is a hearing impairment of more than $20-40 \mathrm{~dB}[25,30]$. With TEOAE screening clicks are presented as stimuli usually at 26 or $36 \mathrm{dBnHL}$ [25].

Use of automated TEOAEs is a convenient screening method. No electrodes are needed and an audiologist is not needed to interpret the results.

Problems with TEOAE screening are that insertion of the probe in the ear canal can be difficult, especially in preterms [24]. The probe must be carefuly fixed and acoustically shielded sealing the outer ear canal. In the newborn hypoxia or infection may result in a reversible reduction in the amplitude spectrum of the TEOAEs [51]. Children with non-organic hearing loss or hearing loss primarily due to involvement of the auditory pathway may have completely normal EOAEs $[3,30]$. Similarly, severe hearing loss due to central nervous system dysfunction or head trauma may show normal EOAEs [3].

Most TEOEA infant testing has been conducted under controlled noise conditions, i.e. in acoustically treated sound rooms or with special procedures such as placing the infant in an isolette [22]. Under these conditions Kennedy et al. [25] found a median time for performing TEAOE screening of $7.2 \mathrm{~min}$ (with 5th and 95th percentiles of $5.8-12.5 \mathrm{~min}$ ) and Stevens et al. [41] found a sensitivity of $76 \%$ and specificity of $86 \%$ when compared to the ABR among the population of a neonatal intensive care unit.

Jacobson and Jacobson [19] carried out TEOAE neonatal hearing screening in a normal hospital environment. Under these conditions the time needed for screening was longer than under strict research conditions. Even though mean recording time for TEOAEs was less than $3 \mathrm{~min}$ for both ears the actual mean time to obtain a result due to noise, myogenic activity, relocation of probes etc. was $16.6 \mathrm{~min}$ (range 7-45 $\mathrm{min}$ ). Under these conditions the specificity and sensitivity of the TEOAE screening was $52 \%$ and $50 \%$ respectively. The noise level measured by the probe microphone within the ear canal exceeded those levels recommended for TEAOE screening in newborns ( $30 \mathrm{dBA}$ SPL). The results of this study suggest that under realistic screening conditions TEOAE results may be influenced by the noise level in the testing environment.

Kemp and Ryan [24] say that in whole population screening it would be quite wrong to suggest to parents that TEOAE screening failure implies a substantial risk of hearing impairment. It simply means the inconvenience of attending for an ABR test. It seems to them more appropriate to regard TEOAE screening failure as an additional risk factor rather than a definitive hearing test.

\section{Stapedius muscle reflex}

The stapedius muscle reflex is a response by muscles of the middle ear to suprathreshold acoustic stimulation. Impedance measurements are made with the insert probe in one ear and the acoustic stimulus (usually 55 or 65 $\mathrm{dBnHL}$ ) is presented to the opposite ear through an ear phone. When a loud sound is presented to one ear the stapedial muscles contract in both ears causing a small change in the acoustic impedance in both ears. This change can be detected by impedance measurement and is proof of the ability of the stimulated ear to code loud noises properly. There is a high rate of false-positive results with this test due to minor middle ear dysfunction prohibiting the detection of the small impedance change. Probe occlusion and head movement also give unreliable results. It is not recommended for preterms. It is not suitable as a screening method because of its low specificity (32\% for the $55 \mathrm{~dB}$ level and $60 \%$ for the $65 \mathrm{~dB}$ level) [38].

Automated measurement of auditory brainstem responses

Until recently there were no viable alternatives to the above mentioned methods available for neonatal hearing screening on a large scale. However, an automated ABR screening method the Algo-1 Plus, (available from Natus Medical Incorporated), has been available since 1985 . This has been developed solely for the purpose of screening for handicapping hearing impairment in young in- 
fants. It is composed of the following elements: an EEG system, a stimulus generating system, ambient noise and myogenic activity detection systems and the ABR detection algorithm. All these components are served by and fed into a central microprocessor. This interprets the outcome of the tests and gives a pass or refer result.

The stimuli used by the Algo-1 Plus are alternating 35 $\mathrm{dB}$ nHL clicks with an acoustic frequency spectrum of $700-5000 \mathrm{~Hz}$ presented monaurally at a rate of 37 pulses/s. The Algo-1 Plus is portable and battery operated. It is very simple to operate and can be used by personnel who have no special audiological training, for instance nursery personnel, child health district nurses or volunteers. It does not need a sound insulated room and can be used in the special care nursery, well baby nursery or the home.

Several clinical trials have been carried out comparing the use of the Algo-1 Plus with the conventional ABR as the gold standard. These show that the Algo-1 Plus compares very favourably with the conventional ABR. Using a controlled protocol Peters [34] found a sensitivity of $100 \%$ and a specificity of $98.7 \%$. In a multi-institutional clinical trial Hall et al. [16] found a sensitivity of $100 \%$ with a specificity $96.7 \%$. Kilney [26] found a sensitivity of $100 \%$ and a specificity $96.15 \%$ and Jacobsen et al. [20] found a sensitivity of $100 \%$ and a specificity of $96 \%$.

The time needed per screening with the the Algo-1 Plus is short. Kennedy found times (mean and 5th and 95th percentiles) of $12.5 \mathrm{~min}$ (8.4-21) for placement of electrodes and $1.5 \mathrm{~min}(0.4-9.8)$ for recording [25]. In our own study of 300 neonates in a hospital setting we found that the mean time needed per screening by volunteers was $19 \min [36]$.

\section{Discussion}

In the study of Martin et al. [29] of deaf 8-year-olds in the EEC the aetiology of the deafness was reported as perceptive hearing loss in $92 \%$, conductive loss in only $2.6 \%$, and $3.8 \%$ had mixed perceptive and conductive loss. In the study of Robertson et al. [39] of children with severe hearing impairment, $75.6 \%$ of cases were due to bilateral sensorineural hearing impairment, $1.5 \%$ were due to congenital conductive loss and $11.7 \%$ due to mixed loss.
When the aim of hearing screening is that infants with serious congenital hearing impairment are to benefit from diagnosis and intervention as early as possible, then the time, money, manpower and other resources used for distraction screening programmes could be put to better use in a neonatal screening programme. Even though distraction testing was introduced to detect children with severe congenital sensorineural hearing loss, these children are usually detected by other methods sometimes earlier, but often later, having "passed" the distraction tests.

The children detected by distraction testing usually have temporary fluctuating conductive hearing loss. If screening for conductive hearing loss is regarded as an aim of screening then the optimum age for this is later than 8-9 months as pointed out by Brown et al. [5] as conductive hearing loss is often part of an ongoing process with relapses and remissions accompanying ear, nose and throat infections during early childhood.

Especially infants with developmental disorders benefit from neonatal hearing screening as behavioural testing can be difficult to interpret in these infants (who also have an increased risk of hearing impairment). When neonatal hearing screening is restricted to those neonates on high risk registers, $33 \%-50 \%$ of children with congenital hearing impairment are undiscovered.

Universal neonatal hearing screening should be the ultimate goal and all neonates should be allowed to benefit from early diagnosis and intervention.

At present two methods are available which could be suitable for large scale neonatal hearing screening: automated measurement of auditory brainstem responses and automated measurement of transient evoked otoacoustic emissions. Large scale studies have been carried out in hospital settings but more trials are necessary to evaluate the practical feasibility and cost- effectiveness of these methods in the home situation. Small scale studies are being carried out by us at present using both these methods in the home. In view of the very high sensitivity and specificity of the automated auditory brain stem method and its ease in use in both hospital and home settings and relatively short time needed per screening we feel that this could be the most effective and practical method for neonatal hearing screening.

\section{References}

1. Baart de la Faille LMB, Kauffman-de Boer MA (1989) Ewing screening and the results (in Dutch). Logopedie en Foniatrie 61: 85-89

2. Barr B, Stensland Junker K, Svard M (1978) Early discovery of hearing impairment: a critical evaluation of the BOEL test. Audiology 17: 62-67
3. Baldwin M, Watkin P (1992) The clinical application of oto-acoustic emissions in paediatric audiological assessment. J Laryngol Otol 106: 301-306

4. Bebout JM (1989) Pediatric hearing aid fitting: a practical overview. Hear J 42: $13-15$
5. Brown $J$, Watson E, Alberman E (1989) Screening infants for hearing loss. Arch Dis Child 64: 1488-1495

6. Cox LC (1984) The current status of auditory brainstem response testing in neonatal populations. Pediatr Res 18: $780-783$ 
7. Curnock DA (1993) Identifying hearing impairment in infants and young children. BMJ 307: 1225-1226

8. De Casper AJ, Fifer WP (1980) Of bonding: newborns prefer their mothers' voices. Science 208: 1174-1176

9. Davis A, Wood S (1992) The epidemiology of childhood hearing impairment: factors relevant to planning of services. Br J Audiol 26: 72-90

10. DHSS Advisory Committee on Services for Hearing Impaired People. Final report of the subcommittee appointed to consider services for hearing impaired children. London HMSO 1981

11. Elliott LL, Armbruster VB (1967) Some possible effects of the delay of early treatment of deafness. J Speech Hear Res 10: 209-224

12. Ewing IR, Ewing AWG (1944) The ascertainment of deafness in infancy and early childhood. J Laryngol Otol 59: 309-333

13. Galambos R, Hicks GE, Wilson MJ (1984) The auditory brain stem response reliably predicts hearing loss in graduates of a tertiary intensive care nursery. Ear Hear 5: 254-260

14. Greenberg MT, Calderon R, Kusche C (1984) Early intervention using simultaneous communication with deaf infants: the effect on communication development. Child Dev 55: 607-616

15. Haggard MP (1990) Hearing screening in children - state of the art(s). Arch Dis Child 65: 1193-1198

16. Hall JW, Kilney PR, Ruth RA, Peters Kripal J (1987) Newborn auditory screening with Algo - 1 vs. conventional auditory brainstem response. Presented at the American Speech-Language-Hearing Association Meeting, New Orleans LA. 15-11-1987

17. Hepper PG, Shahidullah BS (1994) Development of fetal hearing. Arch Dis Child 71: F81-F87

18. Hirasing RA, Dijk C van, WagenaarFisher M, Verveen-Keulemans E, Moulijn-Joustra L, Smith A, Bernts B, Eerdenburg-Keuning I van, Kiers J (1991) Hearing screening in Well Baby Clinics in the Netherlands. Nederlandse Vereniging voor Jeugdgezondheidszorg, NVJG, Utrecht

19. Jacobson JT, Jacobson CA (1994) The effects of noise in transient EOAE newborn hearing screening. Int J Pediatr Otorhinolaryngol 29: 235-248

20. Jacobson JT, Jacobson CA, Spahr RC (1990) Automated and conventional ABR screening techniques in high-risk infants. J Am Acad Audiol 1: 187-195

21. Jewett DL, Romano MN, Williston JS (1970) Human auditory evoked potentials: possible brain stem components detected on the scalp. Science 167: $1517-1518$
22. Johnson MJ, Maxon AB, White KR, Vohr BR (1993) Operating a hospitalbased universal newborn hearing screening program using transient evoked otoacoustic emissions. Semin Hear 14: 46-56

23. Joint Commitee on Infant Hearing (1994) Position statement. ASHA 36: $38-41$

24. Kemp DT, Ryan S (1991) Otoacoustic emission tests in neonatal screening programmes. Acta Otolaryngol (Stockh) [Suppl] 482: 73-84

25. Kennedy CR, Kimm L, Cafarelli Dees D, Evans PIP, Hunter M, Lenton S, Thornton RD (1991) Otoacoustic emissions and auditory brainstem responses in the newborn. Arch Dis Child 66: 1124-1129

26. Kilney PR (1988) New insights on infant ABR hearing screening. Scand Audiol [Suppl] 30: 81-88

27. Kuhl PK, Williams KA, Lacerda F, Stevens KN, Lindblom B (1992) Linguistic experience alters phonetic perception in infants by 6 months of age. Science 255: 606-608

28. Markides A (1986) Age at fitting of hearing aids and speech intelligibility. Br J Audiol 20: 165-167

29. Martin JAM, Bentzen O, Colley JRT Hennebert D, Holm C, Iurato S, Jonge GA de, Mc Cullen O, Meyer ML, Moore WJ, Morgon A (1981) Childhood deafness in the European Community. Scand Audiol 10: 165-174

30. Martin WH, Schwegler JW, Gleeson AL, Yong-Bing Shi (1994) New techniques of hearing assessment. Otolaryngol Clin North Am 27: 487-510

31. Mc Connell F, Liff S (1975) The rationale for early identification and intervention. Otolaryngol Clin North Am 8: $77-87$

32. Mott A, Emond A (1994) What is the role of the distraction test of hearing? Arch Dis Child 70: 10-13

33. NIH Consensus Statement. Early identification of hearing impairment in infants and young children (1993) Bethesda MD: Public Health Service National Institute of Health, Office of Medical Applications of Research 11: $1-24$

34. Peters JG (1986) An automated infant screener using advanced evoked response technology. Hear J 39: 25-30

35. Prager DA, Stone DA, Rose DN (1987) Hearing loss screening in the neonatal intensive care unit: auditory brain stem response versus $\mathrm{Crib}-\mathrm{O}$ Gram; a cost effectiveness analysis. Ear Hear 8: 213-216

36. Oudesluys-Murphy AM, Bholasingh R (1994) Neonatal hearing screening with an automated infant ABR screener. Presented at the VIth International Congress of Pediatric Otorhinolaryngology. Rotterdam. 29 May-1 June 1994
37. Ramkalawan TW, Davis AC (1992) The effects of hearing loss and age of intervention on some language metrics in young hearing-impaired children. $\mathrm{Br}$ J Audiol 26: 97-107

38. Redemann E, Giebel A (1991) Neonatal hearing screening - a necessity in programs for the early detection of impairment. Presented at the 8th International Neonatal Screening Symposium Sydney Australia 12-15 November 1991

39. Robertson C, Aldridge S, Jarman F, Saunders K, Poulakis Z, Oberklaid F (1995) Late diagnosis of congenital sensorineural hearing impairment: why are detection methods failing? Arch Dis Child 72: 11-15

40. Stein LK, Jabaley T, Spitz R, Stoakley D, McGee T (1990) The hearing-impaired infant: patterns of identification and habilitation revisited. Ear Hear 11: 201-205

41. Stevens JC, Webb HD, Hutchinson J, Connell J, Smith MF, Buffin JT (1990) Click evoked otoacoustic emissions in neonatal screening. Ear Hear 11: 128133

42. Stoel-Gammon C, Otomo K (1986) Babbling development of hearing-impaired and normally hearing subjects. J Speech Hear Disord 51: 33-41

43. Tucker SM, Bhatacharya J (1992) Screening of hearing impairment in the newborn using the auditory response cradle. Arch Dis Child 67: 911-919

44. Vademecum Gezondheidsstatistiek Nederland 1992. 's-Gravenhage 1994

45. Van den Brande $\mathrm{Il}$, Gelderen $\mathrm{HH}$ van, Monnens LAH (1990) Kindergeneeskunde Utrecht

46. Van Laar F (1973) Detection of hearing impairment in 9 month old infants (in Dutch). Tijdschr Soc Geneeskd 51: 625-629

47. Veen S, Sassen ML, Schreuder AM, Ens-Dokkum MH, Verloove-Vanhorick SP, Brand R, Grote JJ, Ruys JH (1993) Hearing loss in very preterm and very low birthweight infants at the age of 5 years in a nationwide cohort. Int J Pediatr Otorhinolaryngol 26: $11-$ 28

48. Watkin PM, Baldwin M, McEnery G (1991) Neonatal at risk screening and the identification of deafness. Arch Dis Child 66: 1130-1135

49. White KR, Vohr BR, Maxon AB, Behrens TR, McPherson MG, Mauk GW (1994) Screening all newborns for hearing loss using transient evoked otoacoustic emissions. Int $I$ Pediatr Otorhinolaryngol 29: 203-217

50. Wilson JGM, Jungner G (1968) Principles and practise of screening for disease. WHO, Geneva

51. Zorowka PG (1993) Otoacoustic emissions: a mew method to diagnose hearing impairment in children. Eur J Pediatr 152: 626-634 